A new method of accurate hand- and arm-tracking for small primates

This article has been downloaded from IOPscience. Please scroll down to see the full text article.

2012 J. Neural Eng. 9026025

(http://iopscience.iop.org/1741-2552/9/2/026025)

View the table of contents for this issue, or go to the journal homepage for more

Download details:

IP Address: 134.76.162.165

The article was downloaded on 04/12/2012 at 13:41

Please note that terms and conditions apply. 


\title{
A new method of accurate hand- and arm-tracking for small primates
}

\author{
S Schaffelhofer ${ }^{1}$ and H Scherberger ${ }^{1,2,3}$ \\ ${ }^{1}$ Deutsches Primatenzentrum GmbH, 37077 Göttingen, Germany \\ ${ }^{2}$ Department of Biology, University of Göttingen, D-37077 Göttingen, Germany \\ E-mail: hscherberger@dpz.eu
}

Received 12 August 2011

Accepted for publication 29 November 2011

Published 15 March 2012

Online at stacks.iop.org/JNE/9/026025

\begin{abstract}
The investigation of grasping movements in cortical motor areas depends heavily on the measurement of hand kinematics. Currently used methods for small primates need either a large number of sensors or provide insufficient accuracy. Here, we present both a novel glove based on electromagnetic tracking sensors that can operate at a rate of $100 \mathrm{~Hz}$ and a new modeling method that allows to monitor 27 degrees of freedom (DOF) of the hand and arm using only seven sensors. A rhesus macaque was trained to wear the glove while performing precision and power grips during a delayed grasping task in the dark without noticeable hindrance. During five recording sessions all 27 joint angles and their positions could be tracked reliably. Furthermore, the field generator did not interfere with electrophysiological recordings below $1 \mathrm{kHz}$ and did not affect single-cell separation. Measurements with the glove proved to be accurate during static and dynamic testing (mean absolute error below $2^{\circ}$ and $3^{\circ}$, respectively). This makes the glove a suitable solution for characterizing electrophysiological signals with respect to hand grasping and in particular for brain-machine interface applications.
\end{abstract}

(Some figures in this article are in colour only in the electronic version)

\section{Introduction}

In the past decade, the investigation of cortical motor areas in human and non-human primates has brought new insights into how the brain encodes grasping movements (Baumann et al 2009, Georgopoulos et al 1986, Lemon 2008, Rizzolatti and Luppino 2001, Schieber and Hibbard 1993, Vargas-Irwin et al 2010). However, detailed processes in primary, premotor and parietal areas, and their relationship to hand kinematics are still poorly understood. One reason is the difficulty of tracking hand kinematics of the research field's primary subject, the macaque monkey. So far, grasping tasks have been mainly monitored with sensor-equipped manipulanda (Murata et al 1997, 2000, Schieber 1991), and hand kinematic trackers have been developed only very recently (Overduin

3 Author to whom any correspondence should be addressed.

c)(9) Content from this work may be used under the terms of the Creative Commons Attribution-NonCommercial-ShareAlike 3.0 licence. Any further distribution of this work must maintain attribution to the author(s) and the title of the work, journal citation and DOI. et al 2010, Vargas-Irwin et al 2010). Necessary attributes for hand-tracking systems that solve this problem include high sampling rate, high accuracy, compactness and robustness.

Tracking human hand movements has already been realized successfully for more than 20 years. The DataGlove (VPL Research Inc., Redwood City, CA, USA) (Zimmermann and Lanier 1991) was the first commercially available input glove to gain widespread use in the research community. It was based on optical flex-sensors (Zimmermann 1985) but has been criticized for being unable to measure finger abduction and adduction (MacKenzie 1995, Williams 1997). Current optical gloves, such as the 5DT glove (5DT Inc., Irvine, CA, USA) or the ShapeHand (Measurand Inc. Fredericton, $\mathrm{NB}, \mathrm{CAN}$ ) additionally provide finger abduction/adduction. This results in 14 and 22 degrees of freedom (DOF) of the hand, respectively. Another method for the recording of joint flexion uses electro-resistive sensors (Gentner and Classen 2009, Simone et al 2007, Yun et al 1997) (e.g. CyberGloveII; CyberGlove Systems LCC, San Jose, CA, USA). The resistance of such sensors varies as a function of 


\section{(a)}

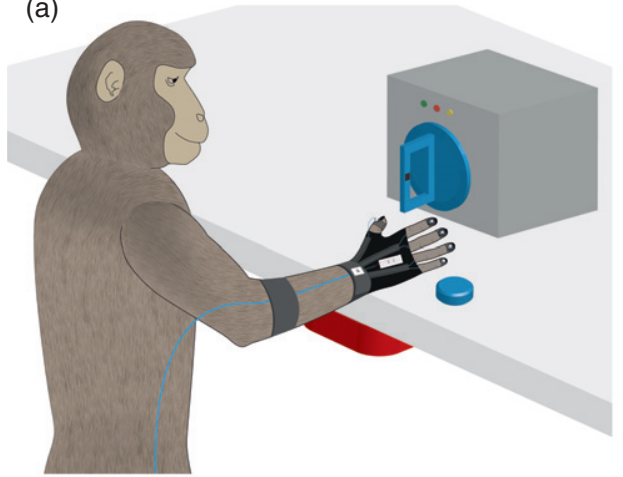

(b)

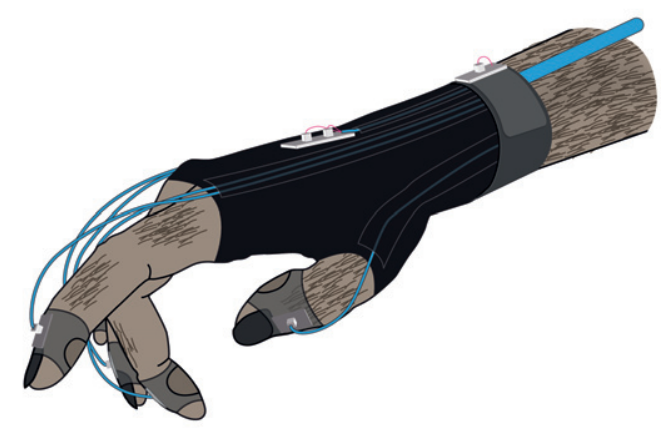

Figure 1. Behavioral setup and tracking glove. (a) Setup. A yellow or green LED instructs the monkey to perform either a precision or a power grip while hand and arm movements are tracked with the instrumented glove. Red LED: eye fixation point. A field generator (red box) placed below the table induces currents in the sensor coils that are proportional to the distance and orientation with respect to the generator. (b) Tracking glove. The tracking system consists of a flexible fingerless glove holding a reference sensor on the hand's dorsum, flexible finger-caps holding a sensor on each fingertip, and micro-tubes guiding the cables and a cable strain relief from the wrist to each fingertip sensor. A wristband holds the arm sensor and fixates the glove with a Velcro (hook-and-loop) fastener.

their bending. Further technology for finger tracking includes Hall sensors (Dipietro et al 2003) that are commercially available as HumanGlove (Humanware S.R.L. Pisa, IT) and force-resistive sensors that allow additional detection of forces to emphasize haptic perception (Castro and Cliquet 1997, Yun et al 1997) (e.g. TouchGlove; Infusion Systems Ltd, Montreal, Canada).

For non-human primates, the first instrumented glove was based on flex sensors (Overduin et al 2010), which is also the most common technology in humans. The glove tracks 9 DOF with nine sensors that are placed onto the desired joints. This type of sensor was evaluated earlier by Simone and Kamper (2005), who found the sensor to be insufficient for sensing the absolute magnitude of bend angles. Also, electroresistive bend sensors are nonlinear and therefore require a time-consuming calibration for each sensor (Overduin et al 2010, Simone et al 2007).

Another recently presented method (Vargas-Irwin et al 2010, Zhuang et al 2010) uses 29 optical markers and 12 infrared cameras to track 25 DOF of the hand and arm of a rhesus monkey. Optical systems provide highly accurate positioning (below $1 \mathrm{~mm}$ ), but markers always rely on line of sight to the cameras. Therefore, the grasping of objects that obscure markers, such as handles or objects with cavities, cannot be tracked.

As a solution for these problems, we present a novel data glove for macaque monkeys based on highly accurate electromagnetic tracking that uses only seven electromagnetic sensors to track 27 DOF of the fingers, hand and arm with a sampling rate of $100 \mathrm{~Hz}$.

\section{Methods}

Animal care and all experimental procedures were conducted in accordance with German and European laws governing animal care and were in agreement with the Guidelines for the Care and Use of Mammals in Neuroscience and Behavioral Research (National Research Council 2003).

\subsection{Glove}

The goal of the glove design was to build a custom-fitted, robust device for daily use with macaque monkeys. Figure 1 shows a schematic drawing of the data glove in the context of the experimental setup and on the animal's hand. Our glove consists of three parts: a wristband, a fingerless glove and fingertip caps (figure 1(b)). The glove holds seven magnetic sensors (see section 2.2. Sensor system and corresponding figure 2), one on each of the five finger caps, one on the hand's dorsum and one on the distal forearm. To ensure tight fitting of the sensors, the finger caps are made of elastic silicon tubes, whereas the fingerless main glove and the wristband are made of spandex (Spandex House Inc., New York, USA). Sensors are held by thin plexiglass plates $\left(7 \times 4 \times 0.8 \mathrm{~mm}^{3}\right)$ that are stitched onto the glove and finger caps. Such a plate avoids possible tilt of the sensors and adapts to the finger shape when stitched onto the elastic silicon tubes. The full glove including sensor cables $(1 \mathrm{~m})$ weighs $16 \mathrm{~g}$. Placement of the glove starts by pulling the elastic glove over the monkey's hand. The glove is adjusted properly and fixed with a Velcro (hook and loop) fastener on the wristband. Then, the elastic fingertip caps are stretched with a sleeving clamp (Facom GmbH, Wuppertal, Germany) and pulled over the animal's fingers.

To achieve high accuracy of fit, a plaster cast model of the monkey's hand was made while the animal was anesthetized for routine examinations. The glove was then fitted to this plaster model.

\subsection{Sensor system}

To track hand and arm movements, we used the electromagnetic tracking system WAVE (Northern Digital, Waterloo, CA). The technology is based on generating nearfield, low-frequency magnetic fields from an assembly of stationary antenna coils (transmitters), which are detected with one sensor coil (5 DOF sensor) or with two sensor coils arranged perpendicular to each other (6 DOF sensor). The field generator (figure 1(a), red box below table) induces currents in the passive receiver coils that are proportional to the strength of 
the current in the transmitter, the distance between transmitter and receiver and the orientation of the receiver. Sequential activation of the transmitter coils results in a sensor output of a set of linearly independent vector fields. This output contains sufficient information to determine the position and orientation of the sensor relative to the transmitter (Bashashati et al 2006, Raab 1982). The carrier frequency of the system is kept proprietary, but measurements show that it operates in the lower $\mathrm{kHz}$ frequency band.

The 5 DOF sensors (dimensions: $3 \times 3 \times 3 \mathrm{~mm}^{3}$ ) provide information on the three-dimensional spatial position $(x, y, z)$ as well as on the yaw and pitch angle with respect to the coordinates of the field generator; 6 DOF sensors (dimensions: $9.5 \times 4.5 \times 4 \mathrm{~mm}^{3}$ ) additionally provide a roll angle. The system allows sensor tracking within a defined measurement volume of $0.5 \times 0.5 \times 0.5 \mathrm{~m}^{3}$ at a sampling rate of $100 \mathrm{~Hz}$. The accuracy of the sensors for static measurements is $0.6 \mathrm{~mm}$ root mean square (RMS) for position and $0.2^{\circ} \mathrm{RMS}$ for orientation, whereas for dynamic tracking the accuracy is $1.5 \mathrm{~mm}$ RMS for position and $0.6^{\circ} \mathrm{RMS}$ for orientation (Northern Digital Inc. 2010).

\subsection{Setup}

Electromagnetic tracking systems do not depend on line of sight but are influenced by the presence of conductive or ferromagnetic materials (Raab et al 1979). For this reason, metal was avoided as much as possible within the setup, on the animal chair and on the grasping manipulandum. Distortions caused by non-ferromagnetic materials such as titanium or stainless steel (DIN 1.441) are negligible (Kirsch et al 2006).

\subsection{Software}

To control the tracking device and for data acquisition, a graphical user interface was implemented in Matlab (The MathWorks Inc., Natick, MA). The software allowed realtime acquisition of the sensor data as well as online modeling of the hand. The computed hand was visualized at up to 25 frames per second (though recording occurred at $100 \mathrm{~Hz}$ ). Time-critical functions were implemented in $\mathrm{C}$ and compiled as Matlab MEX-files. This resulted in an iteration time below $10 \mu \mathrm{s}$. Additional options allowed the user to send data over User Data Protocol or a serial interface. This made the system compatible with a wide range of bio-signal recording systems such as RZ2 (Tucker Davis Technologies, Alachua, FL) or Cerebus (Blackrock Microsystems, Salt Lake City, UT), which facilitated synchronous recordings of hand kinematics and multi-channel electrophysiological signals.

\subsection{Hand and arm model}

The computation of the monkey's hand model was based on six magnetic sensors (figure 2). A reference sensor (6 DOF) was placed on the hand's dorsum and defined the local coordinate system of the hand (S6). In this coordinate system, the positions of metacarpal-phalangeal (MCP) joints (points $A_{1}-A_{5}$ ) were fixed. Their relative position to the reference sensor (S6) was determined either by measuring their distances to the center of the sensor or with a calibration routine as described below. Each fingertip sensor (S1-S5) provided its position and orientation of the distal phalanx in spatial coordinates. From these sensor data, the position of the fingertips $\left(T_{1}-T_{5}\right)$ and the distal interphalangeal (DIP) joints $\left(C_{1}-C_{5}\right)$ was calculated.

The joints of a single finger are anatomically restricted to move within a single plane. Therefore, the proximal interphalangeal (PIP) joint (point $B$ ) is located within this plane and can be calculated when the length of the finger phalanges (line $A B$ and $B C$ ) is known (figure 2(b)). Geometrically, two solutions can be found in the plane for the PIP joint position (defined as $B$ and $B^{\prime}$ in figure 2(b)), but only the case where the PIP joint and the fingertip $T$ lie on opposite sides of the line $A C$ is physiological. From these joint positions, which also determine the orientation of the phalanges, all 20 finger joint angles could be extracted, including flexion, extension of all finger joints (DIP, PIP, MCP) and adduction/abduction (spread) of each finger at the MCP joint.

The modeling of the arm was realized by employing a sensor (S7) on the distal forearm close to the wrist, which measured the orientation of the lower arm. Since the elbow joint is located on the sensor's axis, its location can be calculated if the length of the forearm is known. Finally, we assumed the shoulder position of the animal to be fixed in space; this allowed us to compute a full model of the monkey's arm (i.e. 7 DOF) including the elbow angle and flexion/extension, adduction/abduction and lateral and medial rotation of the shoulder as well as flexion/extension, adduction/abduction and pronation/supination of the wrist.

As mentioned above, the position of the MCP joints relative to the reference sensor can be measured manually. To improve this step, we developed a calibration method that determines the MCP joint positions automatically: when a finger is stretched, the sensor axis points in the direction of the MCP joint. Shifting the position of the fingertip along this axis by the length of the finger determines the position of the MCP joint. To apply this routine, we placed the animal's outstretched hand with the palm down on the table in front of it. The position of the MCP joints was computed for $1 \mathrm{~s}$ (100 samples) and averaged. Resulting MCP positions (relative to the reference sensor) were then used during real-time tracking of the hand but could also be adjusted offline if necessary.

A detailed mathematical description of the hand and arm model and of the calibration routine is presented in the appendix.

\subsection{In vitro evaluation methods}

2.6.1. Static accuracy. To determine the accuracy of the hand-tracking system and the subsequent hand model calculations, we placed the sensor coils on a wooden hand model with five fingers, which could each be moved in 3 DOF. After calibration, the orientations of the proximal phalanx (PPO), middle phalanx (MPO) and distal phalanx (DPO) were systematically altered within their movement range in steps of about $5^{\circ}$, and their corresponding elevation angles recorded with the magnetic sensor system. For verification, a highresolution camera (Canon EOS 550D with lens Canon EF 
(a)

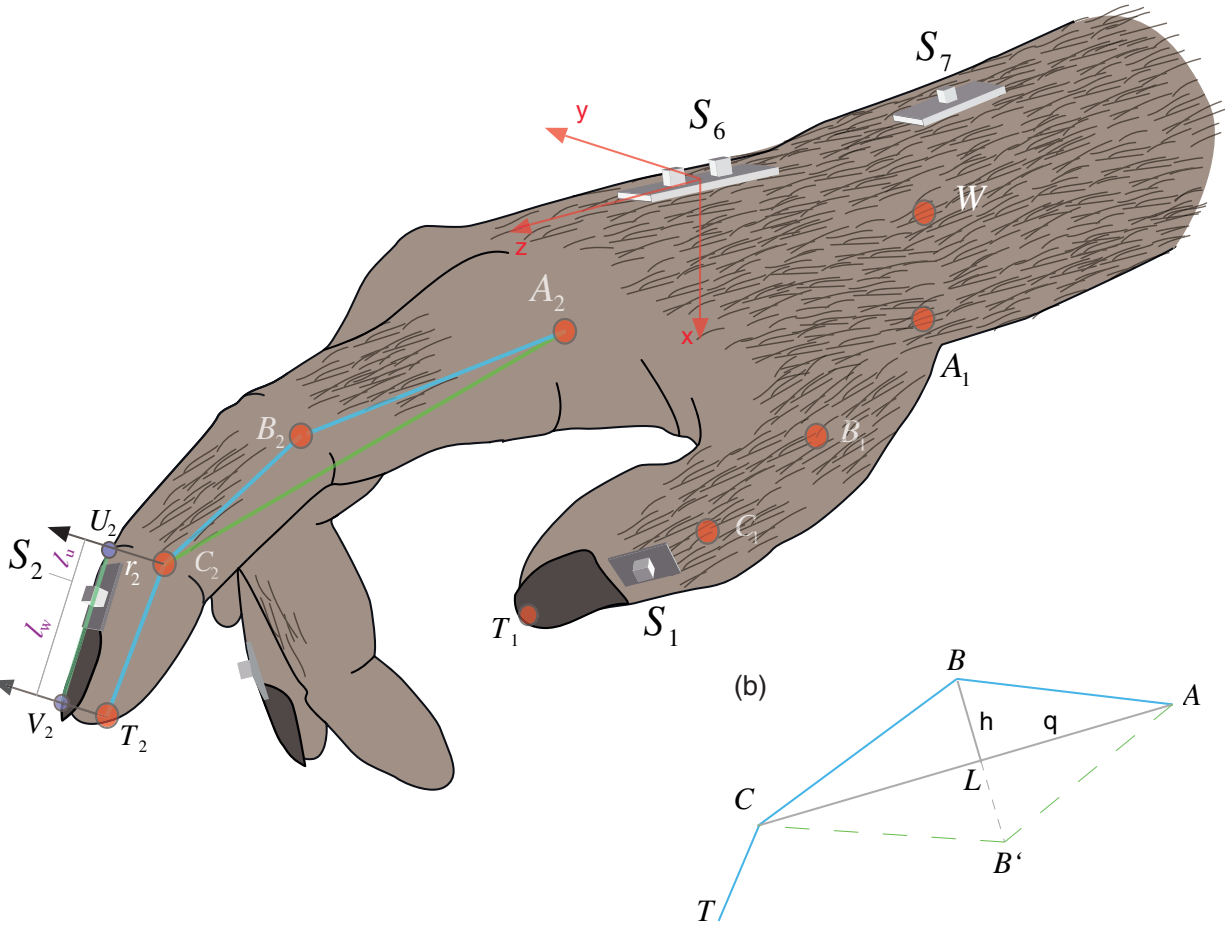

Figure 2. Hand model. (a) Seven magnetic sensors $\left(S_{1}-S_{7}\right)$ define seven points and orientations on the monkey's hand. A reference sensor $\left(\mathrm{S}_{6}\right)$ is placed on the hand's dorsum providing the $x$-, $y$ - and $z$-position in 3D space as well as the hand's yaw, pitch and roll angle (6 DOF). $\mathrm{S}_{6}$ defines the origin and orientation of the local coordinate system of the hand. Sensors $\mathrm{S}_{1}-\mathrm{S}_{5}$ are placed on the fingertips of the hand (only $\mathrm{S}_{1}$ and $\mathrm{S}_{2}$ are shown), providing their position and the orientation of the distal phalanx in order to find points $U$ and $V$ on the finger's surface. The projection of $U$ and $V$ to the distal phalanx center leads to the DIP joint $(C)$ and the fingertip $(T)$. Since $A, C$ and $T$ are moving within the same plane, the PIP joint position $(B)$ is also located on this plane and can be computed. (b) From the measured length of the proximal and middle phalanges $\left(A B\right.$ and $B C$ ) and the calculated distance $(A C)$, two solutions emerge for the PIP joint in the plane: $B$ and $B^{\prime}$. However, the correct solution is the one where $B$ and $T$ are on opposite sides of the line $A C$, which is the physiological case.

70-200 mm) was placed parallel to the hand to take photos of the markers placed on each finger joint (MCP, PIP and DIP) and the fingertip. Images were loaded into Matlab to detect the markers manually for computing all phalanx orientations. The camera resolution of $3456 \times 5184$ pixels allowed a theoretical resolution of $0.02^{\circ}$. Real joint angles, based on the images, were compared with computed angles of the joints (figure 3(a)).

2.6.2. Dynamic tracking. To confirm a sufficiently fast tracking speed for detecting fast reaching and grasping movements of rhesus monkeys, we placed the sensors again on the wooden hand and moved it through the measurement volume with various speeds. We computed the hand model while moving the wooden hand at speeds up to $3.2 \mathrm{~m} \mathrm{~s}^{-1}$. The computed orientations of the phalanges during movements were compared to their static orientation to evaluate the error of movement speed on tracking accuracy (figure 3(b)).

\subsection{In vivo evaluation methods}

2.7.1. Biosignal interference. Developing a device compatible with neural recordings requires testing for possible bio-signal interferences. Bashashati et al (2006) showed that electromagnetic tracking with this device does not disturb EEG recordings within a frequency range of $0.1-55 \mathrm{~Hz}$. However, the potential disturbance on the extracellular recording of action potentials (frequency band: $300-7000 \mathrm{~Hz}$ ) has not yet been investigated.

To evaluate this possible interference, we recorded spiking activity from four implanted floating microelectrode arrays (MicroProbe Inc., Gaithersburg, MD, USA) in a separate animal that had been implanted previously: two arrays had been placed in the ventral premotor cortex (F5) on the bank of the arcuate sulcus, and two further arrays had been implanted in the anterior intraparietal cortex toward the lateral end of the intraparietal sulcus. A detailed description of the electrode arrays and the surgical procedures is presented in Townsend et al (2011). Neural signals were amplified $(300 \times)$ and digitized with 16 bit resolution $\left(0.25 \mu \mathrm{V} \mathrm{bit}{ }^{-1}\right)$ at $30 \mathrm{kS} \mathrm{s}^{-1}$ using a Cerebus Neural Signal Processor (Blackrock Microsystems, Salt Lake City, UT).

Neurons were recorded under four conditions, each lasting 2 min: tracking system disabled (C1) or tracking system enabled, and the field generator placed at a distance of 50 (C2), 30 (C3) or 10 (C4) from the animal's head.

As a first test, we investigated the noise levels of the recordings for different distances (conditions $\mathrm{C} 1-\mathrm{C} 4)$ to evaluate how much the interference affected the quality of the recordings. The noise level $(3 \times$ s.d. $)$ was determined for the continuous and filtered (bandpass $0.3-7 \mathrm{kHz}$ cut-off frequency, Butterworth fourth order) data (figure 4(b)) over 56 channels. 
(a)

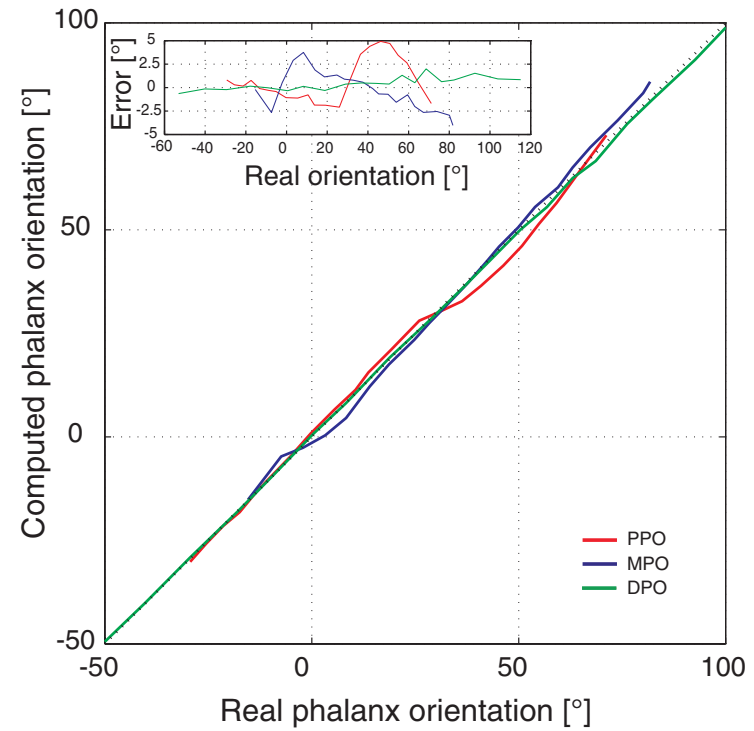

(b)

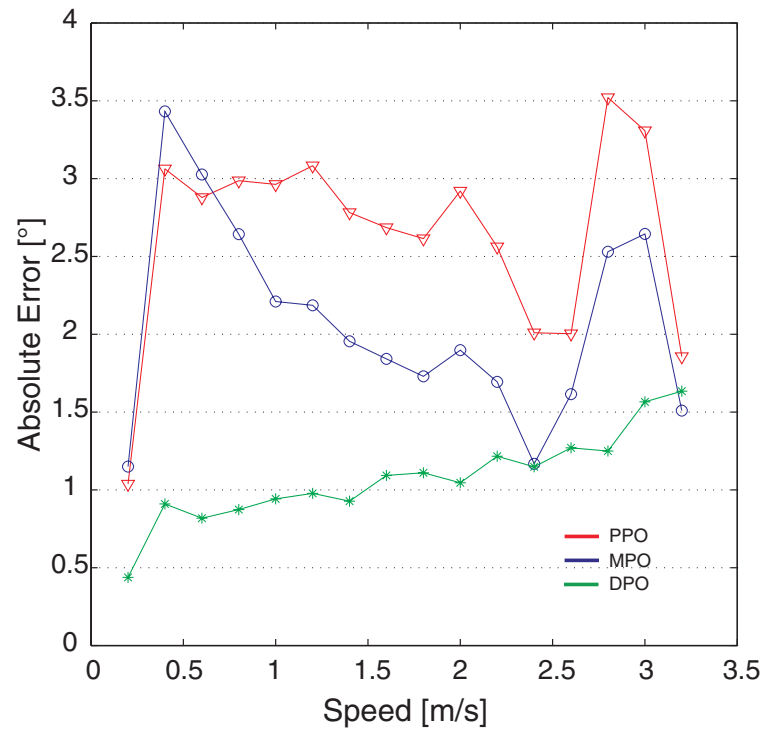

Figure 3. Tracking accuracy. A wooden hand model was used to verify the static and dynamic accuracy of the computed hand model for the PPO, MPO and DPO. (a) Static accuracy. The wooden hand was kept static while altering the orientation of the finger phalanges stepwise over their full range of motion. For every step, the computed phalanx orientation was plotted versus its real orientation, as determined by visual markers and a high-resolution camera. The inset shows the measurement error of the phalanx orientation (real - computed) over the full motion range. (b) Dynamic accuracy. For testing dynamic accuracy, the wooden hand was moved with various speeds while the orientation of all phalanges was kept constant. The measurement error was then defined as the difference between the dynamic and the static orientation of a phalanx. The panel shows the mean absolute error for the three phalanges of a finger (PPO, MPO, DPO) versus the speed of the hand (bin size $=0.2 \mathrm{~m} \mathrm{~s}^{-1}$ ).

(a)

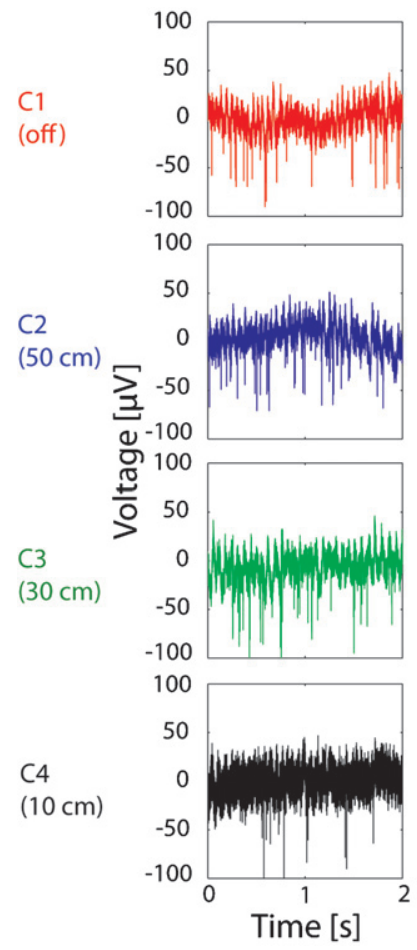

(b)
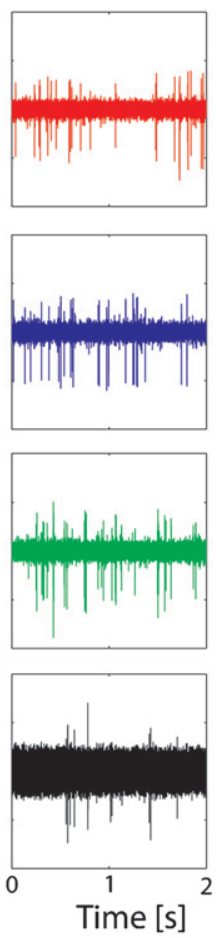

(c)
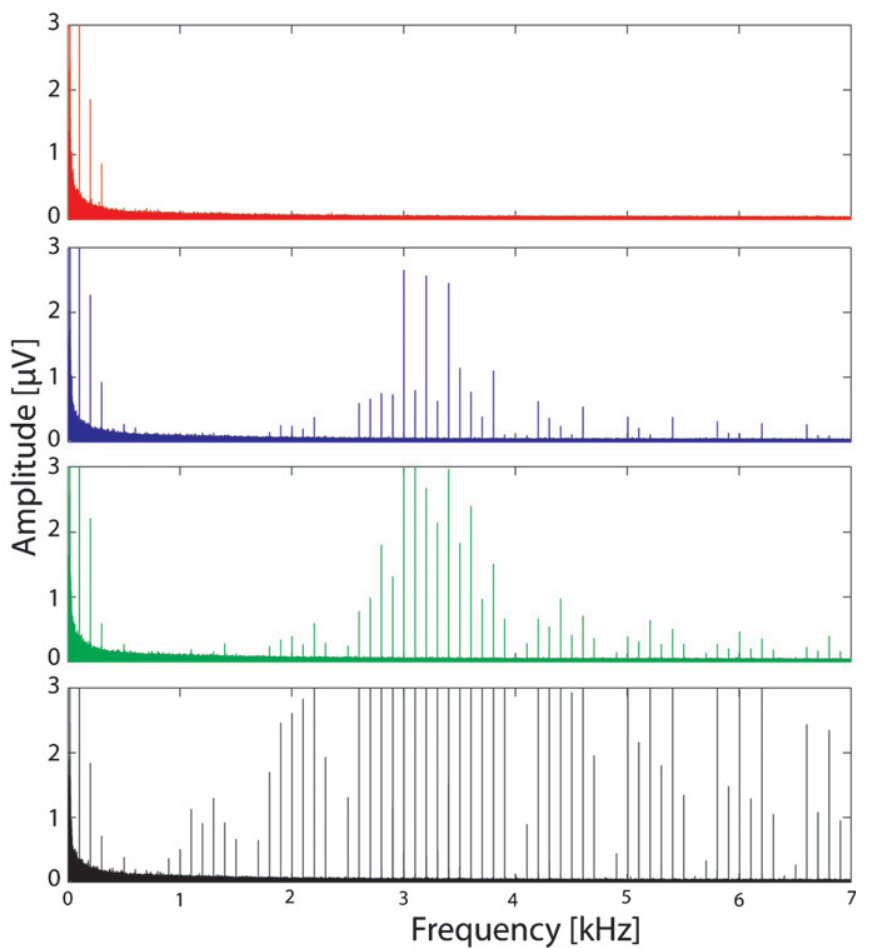

Figure 4. Electrophysiological signal disturbance by the field generator. The effect on extracellular recordings was tested with the device off $(\mathrm{C} 1$, red), and while operating at a distance of $50 \mathrm{~cm}(\mathrm{C} 2$, blue $), 30 \mathrm{~cm}(\mathrm{C} 3$, green $)$ or $10 \mathrm{~cm}(\mathrm{C} 4$, black) from the head of the monkey. (a) Raw, continuous extracellular recordings of an example channel. (b) Same data, bandpass filtered (0.3-7 kHz; fourth-order Butterworth). (c) Amplitude spectrum of the raw extracellular recording. 


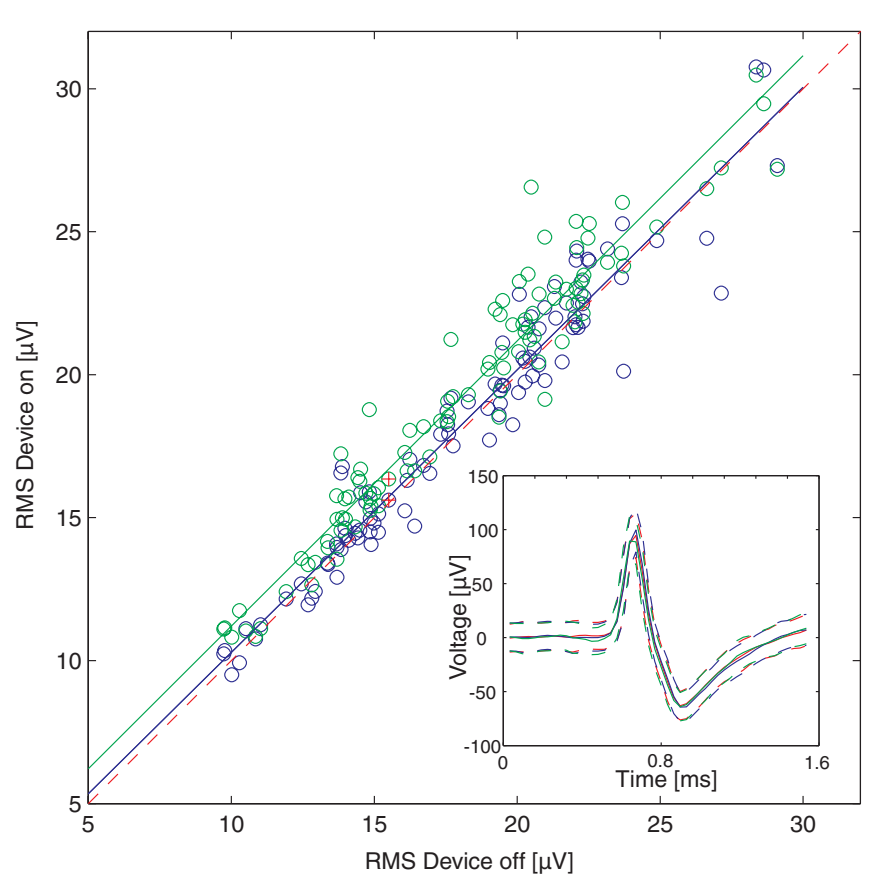

Figure 5. Stability of spike waveforms. 99 neurons were detected on 56 channels under three conditions: (C1) field generator off, $(\mathrm{C} 2)$ head-generator distance $=50 \mathrm{~cm}$, and (C3) head-generator distance $=30 \mathrm{~cm}$. For each condition $\mathrm{C} 1-\mathrm{C} 3$, the waveforms of a unit were subtracted from the mean waveform in $\mathrm{C} 1$ and the residual RMS value calculated over all samples per condition. The RMS value of condition C2 (blue circles) and condition C3 (green circles) is plotted versus that of condition $\mathrm{C} 1$ separately for each neuron. Dashed red line: unity line; blue solid line: least-squares fit of RMS in $\mathrm{C} 2$ versus $\mathrm{C} 1$; green solid line: same for $\mathrm{C} 3$ versus $\mathrm{C} 1$. Inlay: spike shapes of an example unit under conditions $\mathrm{C} 1$ (red), C2 (blue) and C3 (green); solid line: mean spike shape, dashed lines: RMS border of the waveforms.

In a second test, we investigated the possible effects on the quality of the detected waveforms. For this, we compared the waveforms of 99 neurons for condition $\mathrm{C} 1$ against $\mathrm{C} 2-\mathrm{C} 3$. For spike sorting, we used manual and automatic spike-sorting techniques in Plexon-Offline-Sorter (Plexon Inc., Dallas, TX, USA). To determine the possible effects on the waveforms for conditions $\mathrm{C} 1-\mathrm{C} 3$, we first subtracted all waveforms of a unit from its mean waveform under condition $\mathrm{C} 1$ (device off). From this, the RMS value was calculated for each unit separately for conditions C1-C3 (figure 5). The RMS values between these conditions were compared using a paired $t$-test $(p<0.05)$.

\subsubsection{Repeatability. The sensor's repeatability was} evaluated by using a standardized evaluation method (Dipietro et al 2003). To proof the variability in a recording session, the first 100 successful trials were separated into 10 consecutive blocks of 10 trials (Overduin et al 2010). The measured joint angles were time-aligned and averaged over $0.8 \mathrm{~s}$ while the monkey pressed the hand-rest button. An array $\left(X_{i j k}\right), i=$ $1, \ldots, 10, j=1, \ldots, 10, k=1, \ldots, 27$, was finally obtained to specify the data for the $i$ th trial in the $j$ th data block and related to the $k$ th joint angle. For each session, the range was defined as $R_{k}=\left(\max _{j}\left(\bar{X}_{j k}\right)-\min _{j}\left(\bar{X}_{j k}\right)\right)$, where $\bar{X}_{j k}=\frac{1}{10} \sum_{i=1}^{10} X_{i j k}$. In a next step, we calculated the average of $R_{k}$, the standard deviation (s.d.) of the $\bar{X}_{j k}$ values and the average of the s.d. across all joint angles. The average range and the average s.d. across the joint angles were used to define the glove's repeatability.

In an additional test, we computed the repeatability between days. For this, we defined the first 100 correct trials of each day as a distinct block and computed the average range and s.d. across these days. To estimate the day-to-day variability of the MCP positions, we calculated the distance between adjacent MCP joints: $\operatorname{MCP}(k, n)-\operatorname{MCP}(k+1, n)$, ( $k$ : finger number, $n$ : day number). The standard deviation over all days was then averaged for all joints $k$, describing the variability of the MCP estimation.

\subsection{Subject}

Kinematic data were recorded from an 8 year old female rhesus monkey (Macaca mulatta) with a weight of $7.0 \mathrm{~kg}$. In sequential steps, the monkey was trained to tolerate the sensor glove by applying positive reinforcement training techniques (Laule et al 2003, Prescott et al 2005). We started to condition the animal to hold hands for periods of more than $1 \mathrm{~min}$. In the next step, we manipulated the animal's hand with pieces of fabric around its wrist and fingers until the animal tolerated the whole glove. Training duration was about 4 months until the animal tolerated the glove. This time includes extensive testing of the different prototypes of the glove. It should be noted that this first animal was by its nature quite active and distractible. A second, more relaxed animal (not included in this study) could be trained to tolerate the glove in about a month.

For the experiment, we recorded hand kinematics in five daily sessions of about $2 \mathrm{~h}$ duration.

\subsection{Behavioral training}

The animal was trained to perform power or precision grips in a delayed grasping task, as described in detail in previous publications (Baumann et al 2009, Fluet et al 2010) (figure 1(a)). In short, the animal had to place its hand on a capacitive switch to initiate a trial. Then, two LEDs indicated the grip type and the start of the grasping task. Force sensors and push buttons were used to detect power or precision grips. All correct trials were rewarded with a fixed amount of juice, and the animal could initiate the next trial after a short intertrial interval of $500 \mathrm{~ms}$. Error trials were immediately aborted without giving a reward and were followed by a longer intertrial interval (1500-2500 ms). The animal's behavior and all stimulus presentations were controlled using custom-written behavioral control software implemented in LabView Realtime (National Instruments), which allowed a time resolution of $1 \mathrm{~ms}$. 


\section{Results}

\subsection{In vitro testing}

After the assembly of the glove, we first performed in vitro tests to measure the static and dynamic accuracy of the hand model. This was performed on a wooden hand (see section 2.6).

3.1.1. Static accuracy. We used the wooden hand model to evaluate the accuracy of the hand-tracking system. Optical markers were placed on the wooden hand to precisely measure the finger joint angles photographically. Real joint angles were then compared with the computed joint angles from our model (see figure 3(a)). Across all measurements, we found an absolute error of $1.73^{\circ} \pm 2.2^{\circ}$ (mean \pm s.d.) for the PPO, $1.65^{\circ} \pm 2.03^{\circ}$ for the MPO and $0.61^{\circ} \pm 0.66^{\circ}$ for the DPO. This confirmed that the data glove is sufficiently accurate for finger tracking in static (no motion) conditions.

3.1.2. Dynamic tracking. We then used the wooden hand to evaluate the effect of fast movements on measurement accuracies. For this, we brought the hand in a fixed posture and compared the joint angles measured in static position with those measured while the hand was moving with various speeds (figure 3(b)). We found slightly but significantly higher measurement errors when the speed increased (Pearson's correlation coefficient: $r=0.29, p<0.01)$. We measured an absolute error of $0.81^{\circ} \pm 0.84^{\circ}$ (mean \pm s.d.) for DPO, $2.11^{\circ} \pm 3.26^{\circ}$ for MPO and $2.41^{\circ} \pm 3.36^{\circ}$ for PPO. The manufacturer accuracy specifications $\left(0.60^{\circ} \pm 0.7^{\circ}\right)$ could be confirmed, but only for speeds below $0.5 \mathrm{~m} \mathrm{~s}^{-1}$. These errors are generally tolerable, in particular, since they do not accumulate across various finger joints. In other words, the position and orientation of the fingertip are measured directly by the fingertip sensor and are therefore most accurate. Overall, these in vitro results confirmed the suitability of this tracking method for hand and finger tracking in monkeys.

\subsection{In vivo testing}

3.2.1. Usability. Using positive reinforcement training, we trained a macaque monkey to wear the data glove in a primate chair and within the experimental setup. The animal performed a delayed grasping task, which it had previously learned for a different experiment. Wearing the glove did not cause a decline in task performance (with glove: $89.8 \% \pm$ $2.1 \%$ correct trials; without glove: $85 \% \pm 2.3 \%$ ), nor did it reduce the number of trials performed by the animal per session. The hand and arm kinematics of the macaque monkey were recorded over five consecutive days. In total about 6900 grip trials were recorded. The animal performed $1107 \pm$ 77 (mean \pm s.d.) correct trials per day. By comparison, during the five training days prior to the experiments, when the animal was not wearing the glove, it performed an average of $1029 \pm 142$ (mean \pm s.d.) correct trials per day. The monkey tolerated the glove during recording sessions. In addition, the glove and sensors were robust enough to withstand situations in which the monkey tried to remove the glove. The specific glove design allowed attaching the glove to the monkey's hand in 5-10 min before each recording session.

3.2.2. Measurement repeatability. Measurement repeatability addresses the question how much the measured joint angles change within a recording session or between recording days while the animal performed the well-trained grasping task. To allow a comparison of our results with previous work, we used standardized evaluation methods (see section 2.7.2). Following previous investigators (Dipietro et al 2003), we computed the average repeatability range and the s.d. metrics across all joint angles both within a day and between different days. Repeatability within a recording session showed an average range of $5.4^{\circ}$ and an average s.d. of $1.65^{\circ}$ across all computed joint angles. In contrast, between-day repeatability had an average range of $12.7^{\circ}$ and an average s.d. of 5.6 .

Furthermore, we computed the distance between the adjacent MCP joints and their variation across days to estimate the day-to-day variability of the computed MCP joint positions (see section 2), and found, across recording days, an average standard deviation of these distances of $1.3 \mathrm{~mm}$. Together, these results clearly demonstrate a tight and reliable fit of the glove and the sensors to the hand, which is a prerequisite for accurate tracking of hand and finger movements.

3.2.3. Bio-signal interference. Next we tested for a possible influence of the magnetic field generator with electrophysiological recordings. Toward this end, we compared the noise level of electrophysiological recordings and the stability of single-unit waveforms for different testing conditions (head-to-field-generator distances): tracking system disabled ( $\mathrm{C} 1)$ and tracking system enabled with the field generator at a distance of $50 \mathrm{~cm}(\mathrm{C} 2), 30 \mathrm{~cm}(\mathrm{C} 3)$ or $10 \mathrm{~cm}(\mathrm{C} 4)$ from the animal's head. We found a massive signal disturbance for condition $\mathrm{C} 4$ that effectively precluded a clear classification of spikes, but for conditions $\mathrm{C} 1-\mathrm{C} 3$, waveforms could be identified and sorted positively for all 99 neurons tested (see figure 5).

Continuous, bandpass-filtered recordings (figure 4(b)) showed a noise level $(3 \times$ s.d.) of $126.8 \pm 48.9 \mu \mathrm{V}$ (mean \pm s.d.) for condition $\mathrm{C} 4$ (distance: $10 \mathrm{~cm}$ ), $44.0 \pm 10.5 \mu \mathrm{V}$ (mean \pm s.d.) for $\mathrm{C} 3(30 \mathrm{~cm}), 41.9 \pm 9.9 \mu \mathrm{V}$ (mean \pm s.d.) for $\mathrm{C} 2(50 \mathrm{~cm})$ and $41.6 \pm 9.4 \mu \mathrm{V}$ (mean \pm s.d.) for condition $\mathrm{C} 1$ (generator off). $\mathrm{C} 1-\mathrm{C} 4$ showed significantly different noise levels (one-way ANOVA $p<0.01$ ), which could be entirely explained by the increase of $\mathrm{C} 4$ (paired $t$-tests: $\mathrm{C} 1-\mathrm{C} 4, \mathrm{C} 2-$ $\mathrm{C} 4$, C3-C4, each $p<10^{-6}$ ). Importantly, the noise levels in condition $\mathrm{C} 1-\mathrm{C} 3$ were not significantly different (one-way ANOVA for $\mathrm{C} 1-\mathrm{C} 3, p=0.4$ ), which demonstrated that a field generator distance of at least $30 \mathrm{~cm}$ did not strongly affect the electrophysiological noise levels. The amplitude spectrum of the continuous signals pinpoints the interferences of the magnetic field generator to the lower $\mathrm{kHz}$ frequency band (figure 4(c)).

Consistent with these electrophysiological continuous signal measurements, spike classification was precluded in condition $\mathrm{C} 4$, but could be easily done in conditions $\mathrm{C} 1-\mathrm{C} 3$. 
Spike waveform variability measured in RMS showed a nonsignificant RMS increase of $0.2 \mu \mathrm{V}$ between conditions $\mathrm{C} 1$ and C2 (paired $t$-test, $p>0.05$ ) and a significant increase of $1.18 \mu \mathrm{V}$ between $\mathrm{C} 1$ and $\mathrm{C} 3(p<0.05)$. A comparison of RMS values of $\mathrm{C} 2$ and $\mathrm{C} 3$ versus $\mathrm{C} 1$ is illustrated in figure 5 . Although the noise level increased slightly, but significantly, a clear classification of all recorded units was still possible and the waveforms remained unchanged (figure 5, inset). These results demonstrate that single-unit recordings are possible and distortions of waveforms are minimal if the distance of the field generator from the animal's head is at least $30 \mathrm{~cm}$, which could easily be accommodated.

\subsection{Hand grasping kinematics}

The sensor's position and orientation were acquired with a sampling rate of $100 \mathrm{~Hz}$. The model of the hand was updated in real time at this rate (figures 6(b) and (c)). Based on the 18 joint positions, the joint angles (27 DOF) were extracted and plotted in figure 6(a). The computational model of the hand and arm includes, for each finger, the MCP joints (flexion, extension, adduction and abduction), the PIP joints (flexion, extension) and the DIP joints (flexion, extension), and for the wrist and arm, the radiocarpal joint (flexion, extension, adduction, abduction, pronation and supination), the elbow joint (flexion, extension) and the shoulder (flexion, extension, adduction, abduction, and lateral and medial rotation). As we will demonstrate, the full hand and arm motions could be tracked reliably across five daily sessions.

The kinematics of the hand showed significant variation for precision and power grips (figure 6(a)). Both grip types made it necessary to rotate the wrist about $90^{\circ}$ to grasp a vertically oriented handle (see figure 1(a)). During precision grips, this rotation was almost completely accomplished by a rotation of the shoulder, whereas during power grips, the rotation was mainly achieved by a supination of the wrist. The medial rotation and adduction of the shoulder during precision grip were compensated by a strong extension and slight abduction of the wrist. This placed the hand in an optimal position with respect to the handle to perform the precision grip. By comparison, during the power grip, the wrist flexed to allow the fingers to encompass the handle. Other components of the reaching movement, mainly involving the shoulder (extension, adduction) and elbow (extension), were similar for both grip types, mainly because of the identical target location.

Finger movements were also markedly different between the two grip types. The aperture, defined as the distance between the tips of thumb and index finger, illustrated this variation. During power-grip trials (figure 6(a); time $t_{1}$ ), the grip aperture first increased and then decreased as the handle was grasped. In precision-grip trials, by comparison, the grip aperture decreased immediately (figure 6(a); time $t_{2}$ ). For power grip, all digits flexed and extended together, whereas during precision grip, digits 3-5 flexed more than the thumb and the index finger. This behavior ensured enough space for thumb and index finger to perform a precise grip on the handle. Furthermore, fingers touching the handle showed a slight extension of their DIP joints (e.g. thumb during precision grip and middle and ring finger during power grip). This was a likely consequence of the pressure exerted by the fingertips on the handle.

\section{Discussion}

\subsection{Strengths}

In this work, we have demonstrated a novel method for tracking finger hand and arm movements of macaque monkeys based on an instrumented glove with only seven sensors. This technique allows tracking of 27 DOF of finger, wrist, elbow and shoulder joint angles of 18 joints and includes their position in threedimensional space. Our new computational hand model allows reducing the number of tracking sensors by exploiting the anatomical geometry of the primate's hand. To our knowledge, this ratio between DOF and number of sensors is higher than for any other published or commercially available method.

The reduced number of sensors and their miniaturization make the glove design well suited for kinematic hand tracking in small primates. As we have demonstrated, our computational model did not require additional sensors on the upper arm or elbow, nor at intermediate or proximal phalanges of the fingers. This made it possible to separate the glove into parts, which made the fitting of the glove on the animal's hand easy and quick. Furthermore, in full fabric gloves, actions such as wrist or finger flexion often cause the glove to slip over the skin, which would then compromise measurement accuracy (Simone and Kamper 2005). Subdividing the glove into independently moving parts reduces this limitation. In addition, full fabric gloves interfere with tactile sensing. In our design, most parts of the arm and hand are free of fabric to reduce impairment of tactile sensation.

Our behavioral task included precision grips in the dark and was therefore highly dependent on tactile information. Good performance in this task therefore clearly demonstrated the suitability of this glove even for complex grasping tasks. Initially, we built a full fabric glove made out of highly elastic Lycra material as a first prototype. Because of the mentioned disadvantages and the long time needed for placement (up to $15 \mathrm{~min}$ ), our subsequent experience with the fingerless glove was much superior.

Recording of 27 DOF with only seven sensors was also possible because of the employed sensor technology, in which a single sensor coil provided 5 DOF. In comparison, single optical markers can measure only the spatial position (3 DOF). Furthermore, magnetic sensor coils can be tracked without dependence on line of sight. This makes it possible to track hand movements that are not visible to a set of cameras (e.g. grasping of hidden objects or of objects in cavities). In contrast, 3D optical tracking systems depend on line of sight to at least two cameras; however, more typically up to 12 cameras are used (e.g. see Vargas-Irwin et al 2010 or Zhuang et al 2010). This is a strong limitation for many experimental designs. Other technologies that do not depend on line of sight are electro-resistive or optical flex sensors. However, these technologies have other serious disadvantages, 
(a)
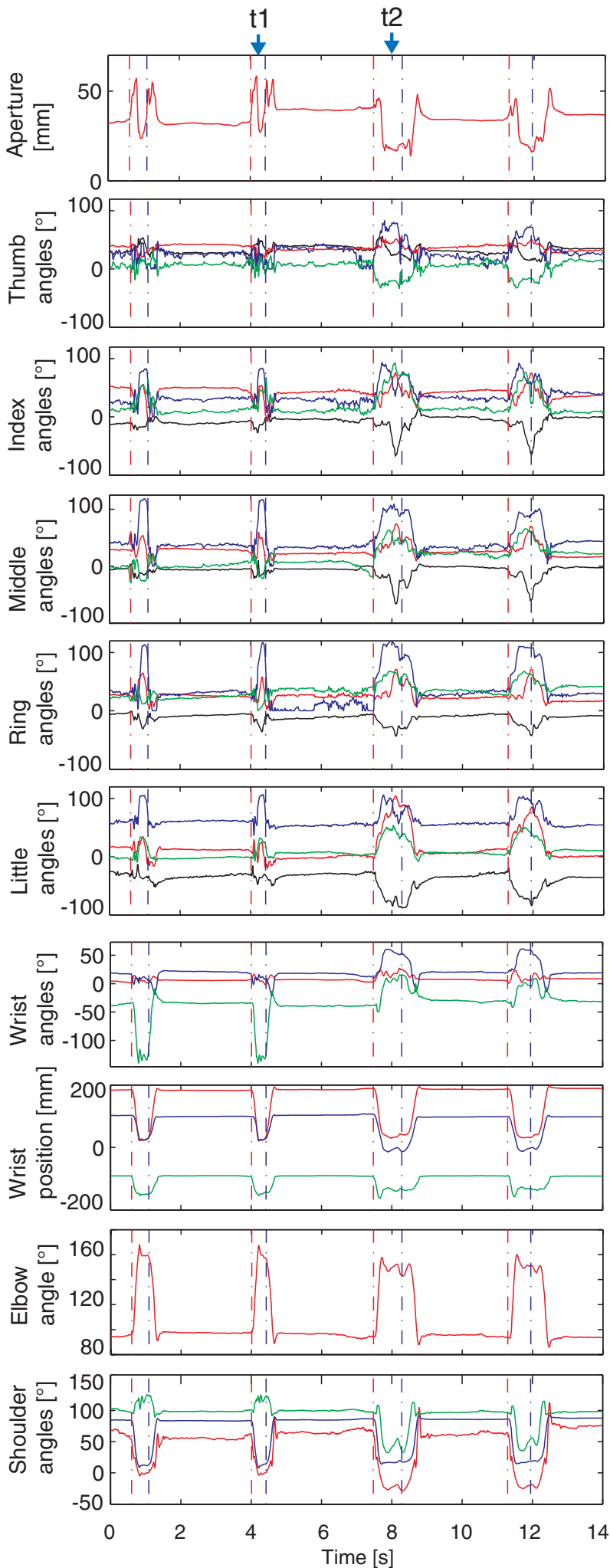

(b)

Power grip (t1)

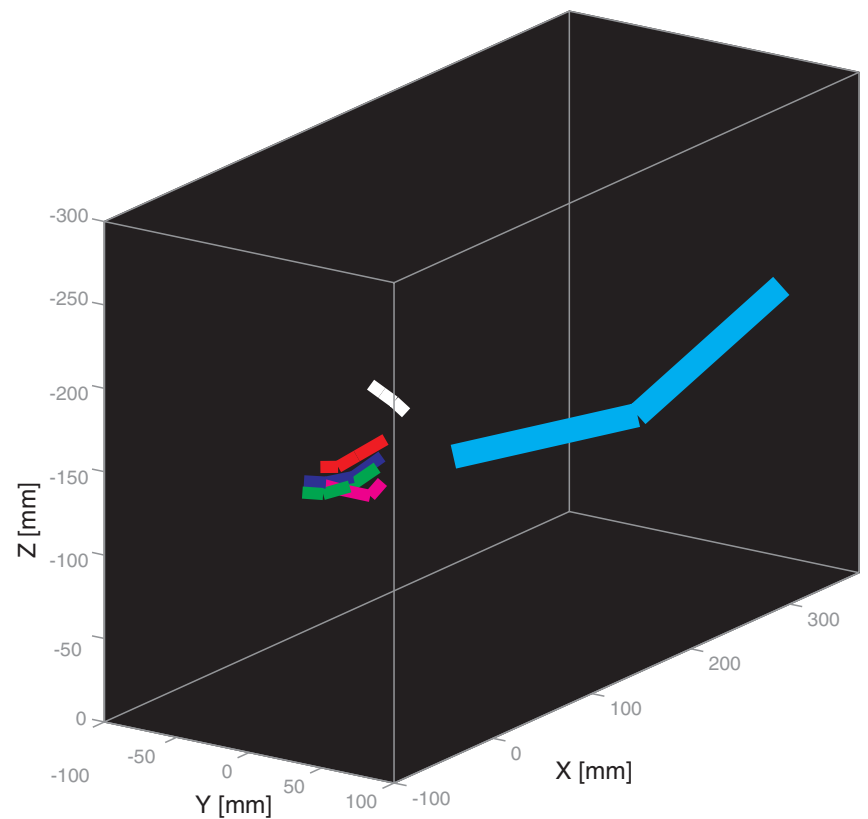

(c)

Precision grip (t2)

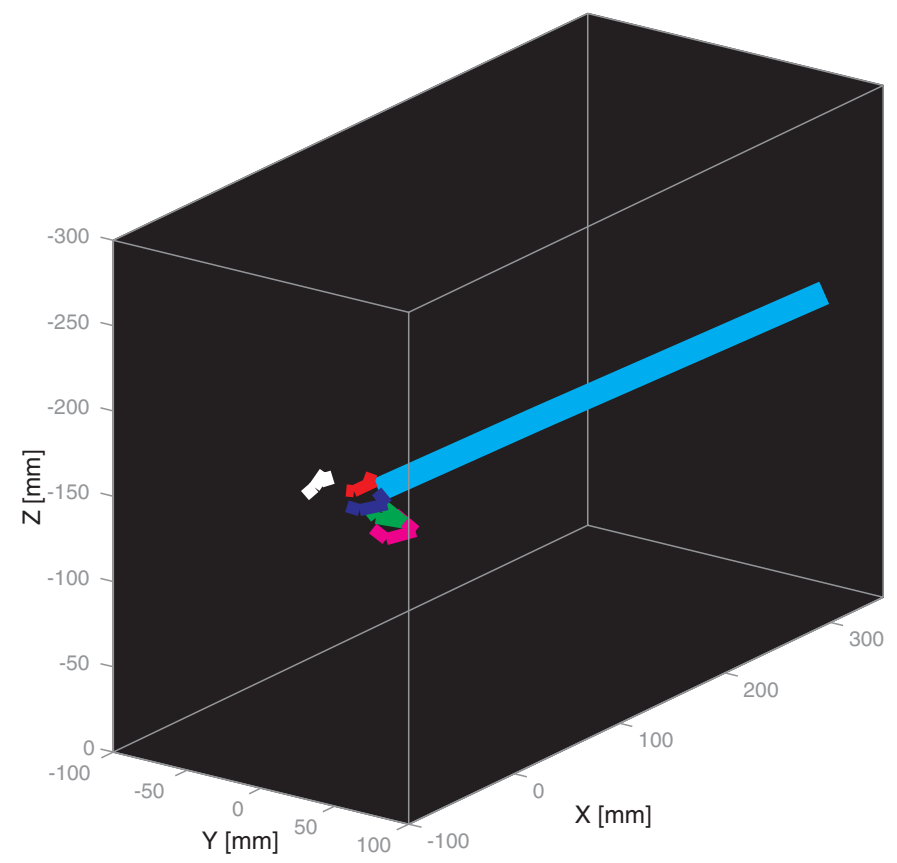

Figure 6. Hand and arm kinematics. (a) Kinematic features of hand and arm are plotted over time. Traces show the performance of two power grips and two precision grips in sequence. Subpanels from top to bottom: hand aperture (red), joint angles of thumb, index, middle, ring and little fingers (MCP in red, PIP in blue, DIP in green, spread in black), wrist orientation (adduction/abduction in red,

flexion/extension in blue, pronation/supination in green), wrist position ( $x$ in red, $y$ in blue, $z$ in green), elbow angle (red) and shoulder orientation (adduction/abduction in red, flexion/extension in blue, lateral/medial rotation in green). The vertical dashed lines indicate the 'go-signal' (red) and the time of reward (blue) for each trial. Screenshot of the real-time computed hand model for power (b) and precision grips (c) at times $t_{1}$ and $t_{2}$, respectively. 
including nonlinearity, the absence of a three-dimensional sensor position and their low accuracy compared to sensor coils (Simone and Kamper 2005).

In our tests, we confirmed the manufacturer's specification for static sensor orientation accuracy of $0.6^{\circ} \mathrm{RMS}$. The same accuracy was also found for many joint angle computations in our model, including the most important parameters for grasping such as flexion/extension of the distal phalanx and the wrist, as well as finger and wrist adduction/abduction. Other parameters for grasp representation, such as MCP and PIP, were measured with lower precision (mean absolute errors $<2^{\circ}$ ) due to the more indirect means of computation; however, these values are still better than the reported values of other methods (Overduin et al 2010). Sensor placement on the distal phalanx, as in our method, can therefore generate highly accurate measurements of fingertip position and orientation, which is particularly advantageous for the study of grip types and hand manipulation.

However, as a precaution, we want to note that in our computational model, as in most others, the palm of the hand was assumed to be stiff (i.e. no palmar flexion). For this reason, the angle errors of the proximal finger angles (MCP, PIP) are expected to be slightly higher for in vivo recordings, while in particular the fingertip position and orientation will be unaffected by this assumption due to the direct measurement of the distal sensor.

Besides the accuracy of the instrumented glove, its robustness is also highly relevant. To guarantee a technology that is reliable and robust enough for applications in macaques, we took several precautions against possible damage. First, we cast the sensors and their cable connections with epoxy resin. Epoxy dries within minutes, protects the sensors and prevents cable movements close to the soldered joints. Second, we used silicon micro-tubes to protect the sensor cables. Furthermore, we passed nylon cords through the micro-tubes parallel to the sensor cables and connected them to the fabric of the glove and the plate carrying the sensors. This provided strain relief for the cables. In our experience, such protection has proved to be sufficient, even though the animal tried several times to remove the glove before getting used to wearing it.

\subsection{Limitations}

Repeatability measurements within a single recording session showed lower variations (range: $5.4^{\circ}$, s.d. $1.65^{\circ}$ ) than for human gloves (range: $7.47^{\circ}$, s.d. $2.44^{\circ}$ ) (Dipietro et al 2003 ) and small primate gloves (range: $7.0^{\circ}$, s.d. $2.3^{\circ}$ ) (Overduin et al 2010). The low within-day variability demonstrates a tight fit of the glove and sensors with no or minimal sensor movements. However, the between-day variability (range: $12.7^{\circ}$, s.d. $5.6^{\circ}$ ) was slightly higher than for human hands (range: $9.38^{\circ}$, s.d. $2.96^{\circ}$ ) (Dipietro et al 2003). This can be explained by the smaller dimensions of the monkey hand, where day-to-day changes of the reference sensor position have a larger effect on the computed angles. To our knowledge, no comparable between-day repeatability measurements have been reported for small primates so far. As a future development, the calibration method could be improved to decrease the between-day error. Instead of determining the MCP joints with the hand stretched out on a table, calibration could be made while the animal grasps a negative mold of its own hand (Dipietro et al 2003), which will likely decrease the variability of the obtained MCP joint positions.

In general, the computational model presented here strongly depends on accurate measurements of limb segments, accurate placement of the sensors and on the digits being represented as hinges centered in the joint space. Using elastic silicon tubes in combination with the sleeving clamp allowed highly accurate positioning of the sensors $(<1 \mathrm{~mm})$. Placing the sensors on thin plastic plates additionally prevented a possible sensor tilt. The silicon tubes ensure a close fit of the sensors to the fingers surface, to get a highly accurate orientation of the distal phalanges. Furthermore, a precise measurement of limb segments is possible, using a plaster cast model of the hand or with MR-imaging. The latter method would additionally allow finding the precise center of rotation of the finger joints, which in primates often lies within the more proximal phalangeal condyle. Furthermore, existing computational methods could be applied for reducing errors caused by sensor noise, inaccurate sensor placement or missing data samples (Todorov 2007).

One of the most relevant disadvantages of an electromagnetic tracking system is distortions of the electromagnetic field by ferromagnetic materials. This makes it necessary to avoid ferromagnetic materials within the setup (e.g. animal chair). However, a stable construction of the experimental setup is still possible with this constraint by using plastics of high density and mechanical strength, such as polyoxymethylene copolymer (tensile modulus = $2700 \mathrm{MPa}$ ), polyetheretherketone (tensile modulus = $3600 \mathrm{MPa}$ ) or glass fiber compounds (e.g. PA 6 GF30, tensile modulus $=9000 \mathrm{MPa}$ ). Metals such as titanium or stainless steel show negligible field distortions and can also be used without constraints (Kirsch et al 2006).

Furthermore, the magnetic field generator of the tracking system could potentially interfere with electrophysiological recordings. The field generator used for this study operated in the lower $\mathrm{kHz}$ frequency band and showed clear superpositions in the extracellular recordings. Increasing the electrodegenerator distance improved the signal quality significantly due to a reduction of the electromagnetic field density, which is proportional to distance squared. Distances $>30 \mathrm{~cm}$ allowed spike sorting without limitation. Fortunately, this distance can easily be accommodated during hand and finger tracking. Furthermore, the amplitude spectrum revealed no interference below $1 \mathrm{kHz}$, which will allow the analysis of local field potentials. For further noise reduction, notch filters could be applied to suppress the field generator's carrier frequency.

The defined measurement volume produced by the field generator was limited to $0.5 \times 0.5 \times 0.5 \mathrm{~m}^{3}$. This is relatively small in comparison to optical systems that allow tracking in a measurement volume of several cubic-meters while providing the same or better spatial resolution. The system's measurement volume was large enough for our purpose, but could limit the design of other tasks (e.g. reaching 
experiments). However, the computational model of the arm extends the effective volume. As shown, the angles of elbow and arm can be computed, even if these body parts are outside the system's measurement volume. If necessary, the measurement volume could be increased by combining the electromagnetic system with other, e.g. optical, tracking systems.

Another limitation of electromagnetic tracking is the relatively low sampling rate, typically around $100 \mathrm{~Hz}$. This is below the frame rates provided by optical systems (up to $1 \mathrm{kHz}$ ). However, even when larger frame rates are recorded, a sampling rate of $\sim 20 \mathrm{~Hz}$ is sufficient to analyze primate hand movements in many cases (Zhuang et al 2010).

\subsection{Possible applications}

The presented hand-tracking device allows the recording of complete hand, finger and arm movements together with electrophysiological signals. This enables a wide range of possible applications for this device. One of the most obvious applications is the investigation of cortical motor areas. Neuronal activity could be correlated with hand and arm kinematics in order to classify them and to understand the neuronal code. Furthermore, the static grip of an object could be compared to the motor plan activity recorded in premotor areas during delayed grasping (Baumann et al 2009, Fluet et al 2010, Townsend et al 2011). As recently shown, instrumented gloves can also be used in the field of BMI to train decoding algorithms in order to reconstruct complete reach and grasp kinematics from neural activity (Vargas-Irwin et al 2010, Zhuang et al 2010). Further applications of the glove might include the investigation of CNS disorders in non-human primates such as spinal cord injury and stroke. In comparison to other systems, the glove presented here measures the position and the orientation of the fingers without depending on line of sight. This advantage allows the detection of abnormal postures, such as twisted and closed hand postures often observed after neurological impairments.

\section{Conclusion}

We have presented a new and reliable technique for finger, hand and arm tracking of primates using a minimal number of sensors. The introduced method is convenient for monitoring reaching and grasping movements online, and it is compatible with electrophysiological recordings. The system shares the main advantages of optical tracking, such as the detection of absolute positions in space but does not depend on line of sight and could therefore be more straightforward than other systems for many neurophysiological applications. The described method is subject to an international patent application.

\section{Acknowledgments}

The authors thank Leonore Burchardt and Natalie Nazarenus for assistance in animal training, Matthias Dörge, Sebastian Lehmann, Jacopo Rigosa and Benjamin Wellner for technical assistance, Lisa Sattlegger for glove production, and Tanya
Lewis, Katharina Menz and Sarah Schwarz for comments on an earlier version of the manuscript. This work was supported by the BMBF (BCCN II, FKZ 01GQ1005C).

\section{Appendix}

\section{Hand model}

Real-time computation of the hand and arm model was based on seven sensors $\left(S_{1}-S_{7}\right)$ placed on the monkey's hand and arm (figure 2). Knowing the position and orientation of the sensors makes it possible to determine, for every finger (1-5), the location of the metacarpal-phalangeal (MCP) joint (points $A_{1}-A_{5}$ ), the proximal-interphalangeal (PIP) joint $\left(B_{1}-B_{5}\right)$, the distal-interphalangeal (DIP) joint $\left(C_{1}-C_{5}\right)$, and the fingertip positions $\left(T_{1}-T_{5}\right)$. Figure 2(a) shows these labels for the thumb $\left(A_{1}, B_{1}, C_{1}, T_{1}\right)$ and the index finger $\left(A_{2}, B_{2}, C_{2}, T_{2}\right)$.

Every sensor $\mathrm{S} \in\left\{S_{1}, \ldots, S_{7}\right\}$ provides its position in Cartesian coordinates $\boldsymbol{s}^{(G)}=\left[x^{(G)}, y^{(G)}, z^{(G)}\right]^{T}$ and its orientation in quaternions $\boldsymbol{q}^{(G)}=\left[q_{0}, q_{1}, q_{2}, q_{3}\right]^{T}$ with respect to the global (field generator) coordinate system $C_{G}$. In addition, each sensor has its own coordinate system $C_{S}$. In $C_{S}$, a point $\boldsymbol{p}$ is defined by the position vector $\left[x^{(S)}, y^{(S)}, z^{(S)}\right]^{T}$ or in homogeneous coordinates: $\boldsymbol{p}^{(S)}=\left[x^{(S)}, y^{(S)}, z^{(S)}, 1\right]^{T}$. This point can be transformed to the global coordinate system $C_{G}$ by the matrix equation

$$
\boldsymbol{p}^{(G)}=M_{S G}^{-1} \boldsymbol{p}^{(S)},
$$

where $M_{S G}$ is the transformation matrix

$M_{S G}=$

$\left(\begin{array}{cccc}q_{0}^{2}+q_{1}^{2}-q_{2}^{2}-q_{3}^{2} & 2\left(q_{1} q_{2}-q_{0} q_{3}\right) & 2\left(q_{0} q_{2}+q_{1} q_{3}\right) & x \\ 2\left(q_{1} q_{2}+q_{0} q_{3}\right) & q_{0}^{2}-q_{1}^{2}+q_{2}^{2}-q_{3}^{2} & 2\left(q_{2} q_{3}-q_{0} q_{1}\right) & y \\ 2\left(q_{1} q_{3}-q_{0} q_{2}\right) & 2\left(q_{0} q_{1}+q_{2} q_{3}\right) & q_{0}^{2}-q_{1}^{2}-q_{2}^{2}+q_{3}^{2} & z \\ 0 & 0 & 0 & 1\end{array}\right)$

and $\boldsymbol{s}=[x, y, z]^{T}, \boldsymbol{q}=\left[q_{0}, q_{1}, q_{2}, q_{3}\right]^{T}$ are the sensor position and orientation in global coordinates $C_{G}$, respectively.

Specifically, a 6 DOF sensor $\left(S_{6}\right)$ defines the local coordinate system $C_{L}=C_{S_{6}}$ on the hand's dorsum, with which the matrix transformation $M_{L G}$ is associated that transforms points from global $\left(C_{G}\right)$ to local coordinates $\left(C_{L}\right)$. For every finger, the position A of the MCP joint is assumed to be constant in $C_{L}$ and can be measured directly or determined by a calibration procedure (see below).

To determine the remaining points $B, C$ and $T$ for each finger, we first defined the points $U^{(S)}=\left[0,0,-l_{U}, 1\right]^{T}$ and $V^{(S)}=\left[0,0, l_{V}, 1\right]^{T}$ in sensor coordinates, where $l_{U}$ and $l_{V}$ denote the distance of the sensor $\mathrm{S}$ along the sensor axis to the DIP joint and the fingertip, respectively; see figure 2(a). These points are then transformed to global coordinates $U^{(G)}=M_{S G}^{-1} U^{(S)}, V^{(G)}=M_{S G}^{-1} V^{(S)}$ and from there to the local coordinates of the hand $\left(C_{L}\right)$ :

$$
U^{(L)}=M_{L G} U^{(G)}, \quad V^{(L)}=M_{L G} V^{(G)} .
$$

Thereby, $M_{L G}$ denotes the transformation matrix from global $C_{G}$ to local coordinates $C_{L}$.

To determine the position of the DIP joint $C$ and the fingertip $T$, points $U$ and $V$ are shifted orthogonally to the sensor axis to the finger's center. To compute this, we 
note that points $A, U$ and $V$ are anatomically restricted to move within a plane (see figure 2). A unit vector normal to this plane is $\boldsymbol{e}(\overrightarrow{A U} \times \overrightarrow{U V})$, where $\boldsymbol{e}(\boldsymbol{x})=\boldsymbol{x} /\|\boldsymbol{x}\|$ denotes the unit vector pointing in the direction of vector $x$. Then $\boldsymbol{n}=\boldsymbol{e}((\overrightarrow{A U} \times \overrightarrow{U V}) \times \overrightarrow{U V})$ lies within this plane, and is orthogonal to $U V$ and has length 1 . From this and the distal radius $r$ of the finger, $C$ and $T$ can be calculated as

$$
\begin{gathered}
C=U+r \boldsymbol{n}, \\
T=V+r \boldsymbol{n} .
\end{gathered}
$$

Finally, to determine point $B$, we consider the triangle $(A B C)$. Points $A$ and $C$ as well as the lengths of the proximal phalanx $(A B)$ and middle phalanx $(B C)$ are known (figure 2(b)). Using the law of cosines, the angle $\alpha$ between $(A B)$ and $(A C)$ follows as $\alpha=\arccos \left(\frac{(A B)^{2}+(A C)^{2}-(B C)^{2}}{2|A B||A C|}\right)$. From this, the orthogonal projection of $B$ on the line $A C$, which we call $L$, can be computed as

$$
L=A+q e(\overrightarrow{A C}),
$$

where $q=\cos (\alpha)|A B|$ gives the distance of $A$ to $L$. Furthermore, $h=\sin (\alpha)|A B|$ notes the length of the line $(B L)$. Together with the unit vector $\boldsymbol{m}=$ $e(\overrightarrow{A C} \times(\overrightarrow{A C} \times \overrightarrow{C T}))$, which lies in the plane $A C T$ and normal to $A C$, we can obtain $B$ as

$$
B=L+h \boldsymbol{m} .
$$

This definition of $B$ for the PIP joint location (instead of the alternate solution $B^{\prime}=L-h \boldsymbol{m}$ ) ensures that the PIP joint and the fingertip position $T$ are always on opposite sides of the line $A C$, as is the case during naturally occurring finger movements.

\section{Arm model}

The computation of the subject's arm position is realized with the sensor $\mathrm{S}_{6}$ on the hand dorsum and sensor $\mathrm{S}_{7}$ on the glove wristband (see figure 2(a)). To create the arm model, the position of the wrist joint $W^{(L)}=\left[x^{(L)}, y^{(L)}, z^{(L)}, 1\right]^{T}$ is determined by manual measurement and transformed to global coordinates $C_{G}$ by

$$
W^{(G)}=M_{L G}^{-1} W^{(L)} .
$$

Then, $W^{(G)}$ and the orientation $\boldsymbol{q}_{7}^{(G)}=\left[q_{7,0}, q_{7,1}, q_{7,2}, q_{7,3}\right]$ of the sensor $\left(\mathrm{S}_{7}\right)$ define a new coordinate system $C_{W}$ of the wrist and the associated transformation matrix $M_{W G}$ that transforms points from global $\left(C_{G}\right)$ to wrist coordinates $\left(C_{W}\right)$.

In $C_{W}$, the position of the wrist joint is the origin $W^{(W)}=[0,0,0,1]^{T}$ and the elbow joint position is given by $E^{(W)}=\left[0,0,-l_{e}, 1\right]^{T}$, where $l_{e}$ is the distance of the wrist joint to the elbow joint. Wrist and elbow positions can be transformed to global coordinates $C_{G}$ by

$$
\begin{aligned}
& W^{(G)}=M_{W G}^{-1} W^{(W)} \\
& E^{(G)}=M_{W G}^{-1} E^{(W)} .
\end{aligned}
$$

Finally, the shoulder position $H^{(G)}$ is assumed to be fixed in space and can be measured directly. This completes the arm model consisting of the global shoulder $(H)$, elbow $(E)$ and wrist $(W)$ positions.

\section{Calibration}

To avoid measuring local distances between sensor $\mathrm{S}_{6}$ and the MCP joints (point A) for every finger, we developed an online calibration method. The monkey was trained to press its hand onto a flat surface with all fingers extended. The surface was arranged orthogonally to the field generator's $z$-axis that points downward. Then for each finger, we defined $K^{(S)}=\left[0,0,-\left(|A B|+|B C|+l_{U}\right), 1\right]^{T}$ as the point along the axis of the fingertip sensor $\mathrm{S}$ just on top of $\mathrm{A}$. This point was transformed first to global coordinates $K^{(G)}=M_{S G}^{-1} K^{(S)}$ and then shifted toward the palm (along the $z$-axis) by finger radius $r$, which led to the MCP joint position in global coordinates $A^{(G)}=K^{(G)}+[0,0, r, 1]^{T}$ and in local coordinates $C_{L}$ of the hand: $A^{(L)}=M_{L G} A^{(G)}$.

\section{References}

Bashashati A, Noureddin B, Ward R K, Lawrence P D and Birch G E 2006 An experimental study to investigate the effects of a motion tracking electromagnetic sensor during EEG data acquisition IEEE Trans. Biomed. Eng. 53 559-63

Baumann M A, Fluet M C and Scherberger H 2009 Context-specific grasp movement representation in the macaque anterior intraparietal area J. Neurosci. 29 6436-48

Castro M C and Cliquet A Jr 1997 A low-cost instrumented glove for monitoring forces during object manipulation IEEE Trans. Rehabil. Eng. 5 140-7

Dipietro L, Sabatini A M and Dario P 2003 Evaluation of an instrumented glove for hand-movement acquisition J. Rehabil. Res. Dev. 40 179-89

Fluet M C, Baumann M A and Scherberger H 2010 Context-specific grasp movement representation in macaque ventral premotor cortex J. Neurosci. 30 15175-84

Gentner R and Classen J 2009 Development and evaluation of a low-cost sensor glove for assessment of human finger movements in neurophysiological settings $J$. Neurosci. Methods 178 138-47

Georgopoulos A P, Schwartz A B and Kettner R E 1986 Neuronal population coding of movement direction Science 233 1416-9

Kirsch S R, Schilling C and Brunner G 2006 Assessment of metallic distortions of a electromagnetic tracking system Proc. SPIE 6141 61410J-1-9

Laule G E, Bloomsmith M A and Schapiro S J 2003 The use of positive reinforcement training techniques to enhance the care, management, and welfare of primates in the laboratory $J$. Appl. Anim. Welfare Sci. 6 163-73

Lemon R N 2008 Descending pathways in motor control Annu. Rev. Neurosci. 31 195-218

MacKenzie I S 1995 Input devices and interaction techniques for advanced computing Virtual Environments and Advanced Interface Design ed W Barfield and T A Furness (New York: Oxford University Press) pp 437-70

Murata A, Fadiga L, Fogassi L, Gallese V, Raos V and Rizzolatti G 1997 Object representation in the ventral premotor cortex (area F5) of the monkey J. Neurophysiol. 78 2226-30

Murata A, Gallese V, Luppino G, Kaseda M and Sakata H 2000 Selectivity for the shape, size, and orientation of objects for grasping in neurons of monkey parietal area AIP J. Neurophysiol. 83 2580-601

National Research Council 2003 Guidelines for the Care and use of Mammals in Neuroscience and Behavioral Research (Washington, DC: National Academies Press) 
Northern Digital Inc. 2010 The wave tracking system Company Brochure

Overduin S A, Zaheer F, Bizzi E and d'Avella A 2010 An instrumented glove for small primates J. Neurosci. Methods 187 100-4

Prescott M J, Bowell V A and Buchanan-Smith H M 2005 Training laboratory-housed non-human Primates: part 2. Resources for developing and implementing training programs Anim. Welfare $4133-48$

Raab F H 1982 US Patent No. 4346348

Raab F H, Blood E B, Steiner T O and Jones H R 1979 Magnetic position and orientation tracking system IEEE Trans. Aerosp. Electron. Syst. 15 709-18

Rizzolatti G and Luppino G 2001 The cortical motor system Neuron 31 889-901

Schieber M H 1991 Individuated finger movements of rhesus monkeys: a means of quantifying the independence of the digits J. Neurophysiol. 65 1381-91

Schieber M H and Hibbard L S 1993 How somatotopic is the motor cortex hand area? Science 261 489-92

Simone L K and Kamper D G 2005 Design considerations for a wearable monitor to measure finger posture J. Neuroeng. Rehabil. 25

Simone L K, Sundarrajan N, Luo X, Jia Y and Kamper D G 2007 A low cost instrumented glove for extended monitoring and functional hand assessment $J$. Neurosci. Methods $160335-48$

Todorov E 2007 Probabilistic inference of multijoint movements, skeletal parameters and marker attachments from diverse motion capture data IEEE Trans. Bio-Med. Eng. 54 1927-39

Townsend B R, Subasi E and Scherberger H 2011 Grasp movement decoding from premotor and parietal cortex J. Neurosci.: Official J. Soc. Neurosci. 31 14386-98

Vargas-Irwin C E, Shakhnarovich G, Yadollahpour P, Mislow J M, Black M J and Donoghue J P 2010 Decoding complete reach and grasp actions from local primary motor cortex populations J. Neurosci. $309659-69$

Williams N W 1997 The virtual hand. The Pulvertaft Prize Essay for 1996 J. Hand Surg. (Br.) 22 560-7

Yun M H, Cannon D, Freivalds A and Thomas G 1997 An instrumented glove for grasp specification in virtual-reality-based point-and-direct telerobotics IEEE Trans. Syst. Man Cybern. B 27 835-46

Zhuang J, Truccolo W, Vargas-Irwin C and Donoghue J P 2010 Decoding 3-D reach and grasp kinematics from high-frequency local field potentials in primate primary motor cortex IEEE Trans. Biomed. Eng. 57 1774-84

Zimmermann T G 1985 US Patent No. 4542291

Zimmermann T G and Lanier J Z 1991 US Patent No. 4988981 